\title{
¿Empoderamiento o Subyugación de la Mujer? Experiencias de Cosificación Sexual Interpersonal
}

\section{Is Empowerment or Women's Subjugation? Experiences of Interpersonal Sexual Objectification}

\author{
Gemma Sáez, Inmaculada Valor-Segura y Francisca Expósito \\ Universidad de Granada, España
}

\begin{abstract}
Resumen. La cosificación sexual interpersonal es la reducción de una mujer a su cuerpo o partes de éste. Este fenómeno puede ser considerado como una forma de discriminación sexista, en la que una mujer es tratada de manera diferente minimizando la importancia de sus atributos internos. Este estudio, en el que se incluyeron 251 participantes de población general, tiene como objetivo analizar el fenómeno de la cosificación sexual interpersonal en hombres y mujeres, y su relación con variables ideológicas (sexismo y poder), autoestima y disfrute de la sexualización. Los resultados mostraron diferencias de género en la cosificación sexual interpersonal, de modo que las mujeres experimentaron en mayor medida experiencias de cosificación en sus relaciones interpersonales. Además, se observó un patrón diferencial de las variables relacionadas con las experiencias de cosificación en función del género. En hombres, la autoestima y el poder fueron variables relacionadas con una mayor percepción de cosificación. En concreto, el poder predice una mayor percepción de cosificación y esta relación estuvo mediada por el disfrute de la sexualización. En las mujeres, es el sexismo benévolo el que lleva a una mayor percepción de cosificación sexual interpersonal y esta relación estuvo mediada por el disfrute de la sexualización.

Palabras clave: autoestima, cosificación sexual, disfrute de la sexualización, poder, sexismo.
\end{abstract}

\begin{abstract}
Interpersonal sexual objectification is defined as the act of reducing a woman to her body or body parts. It could be considered as a form of sexist discrimination, in which women have a differential treatment minimizing the importance of their inner qualities. The aim of this study, in which involved 251 participants, was to analyze the perception of interpsersonal sexual objectification in men and women. In addition, we examined the relationship between the interpersonal sexual objectification and ideological variables (sexism and power) as well as self-esteem and enjoyment of sexualization. Results showed gender differences in interpersonal sexual objectification. Women experienced more sexual objectification in their interpersonal relationships. Also, results showed the effect of gender in the variables that predicted interpersonal sexual objectification. In men, self-esteem and power were related with more experiences of sexual objectification. Specifically, power predited the perception of objectification and this effect was mediated by enjoyment of sexualization. However, in women, benevolent sexism predicted the perception of interpersonal sexual objectification and this effect was mediated by enjoyment of sexualization.

Keywords: enjoyment of sexualization, power, self-esteem, sexism, sexual objectification.
\end{abstract}

En los últimos años, el cuidado de la imagen y el culto al cuerpo se ha convertido en uno de los aspectos más importantes en la vida de las personas. Este interés por la imagen se ha extendido también entre los hombres. Podríamos decir que el cuerpo y la imagen se han convertido en un bien al alza que mueve muchos intereses y que condiciona las relaciones interpersonales. Los países occidentales han asumido esta cultura de forma generalizada llegando incluso a vivir esclavizados por "el culto al cuerpo". España, concretamente, es el primer país europeo en operaciones de estética y el cuarto del mundo. El 8\% de las operaciones de ciru-

Correspondencia: Gemma Sáez Díaz. Departamento de Psicología Social, Facultad de Psicología. Universidad de Granada. Campus de Cartuja,s/n. 18071 Granada (España). E-mail: gemmasaez@correo. ugr.es gía estética se realizan en España, encontrándonos por debajo de EE.UU. (13\%), México (9\%) y Argentina (8.5\%) (Sociedad Española de Cirugía Plástica, Reparadora y Estética, 2006).

La preocupación por el cuerpo y la imagen ha sido un aspecto íntimamente relacionado con la vida de las mujeres. Desde las teorías sociobiológicas, la imagen de la mujer ha sido central para consolidar sus roles sociales (esposa y madre). El valor de una mujer estaba determinado en parte por su juventud y su belleza, aspectos que se relacionaban positivamente con la percepción de fertilidad y que por tanto la convertían en objeto de deseo y muy valorada por los hombres (Sevillano, Rodríguez, Olarte y Lahoz, 2005). No obstante, la relación que la mujer ha mantenido con su cuerpo no siempre ha traído consecuencias positivas. El desarrollo y los cambios sociales que han modifica- 
do los principales roles de las mujeres actualmente, no han llevado asociado un menor interés por el aspecto físico de éstas. La relación que las mujeres tienen con su cuerpo, afecta a su vida personal, profesional y política (Wolf, 1991), y esta relación está influida de manera perniciosa por la cosificación sexual, referida al trato que la sociedad da a la mujer como un objeto sexual, incrementado en los últimos años (Kaschak, 1992).

\section{Cosificación Sexual Interpersonal}

La cosificación sexual es la reducción de una mujer en su cuerpo o partes de éste con la percepción errónea de que su cuerpo o partes del mismo pueden representarla en su totalidad (Bartky, 1990). La cosificación se produce cuando se separan las funciones o partes sexuales de una mujer de su persona, instrumentalizándola o reduciéndola a dichas partes sexuales (Fredrickson y Roberts, 1997). Desde las teorías feministas se ha puesto énfasis en resaltar la importancia que la construcción social tiene en la imagen que las mujeres desarrollan y construyen de sí mismas. Esta idea ha sido recuperada y desarrollada por la Teoría de la Cosificación (Objectification Theory) formulada por Fredrickson y Roberts en 1997. La teoría de la cosificación (TC), subraya la importancia de las experiencias de socialización de género, en concreto, aquellas experiencias que exponen a las mujeres a ser valoradas exclusivamente por su cuerpo. El punto central de esta teoría es la gran presencia de la cosificación sexual de las mujeres en la sociedad y cómo afecta a su bienestar, físico, psicológico y social (Fredrickson y Roberts, 1997). La continua exposición a situaciones en las que las mujeres son sexualmente cosificadas, hace que éstas se perciban a sí mismas como objetos, interiorizando la mirada de un observador externo. Este fenómeno se denomina autocosificación, y se manifiesta por la autovigilancia entendida como "un continuo seguimiento de la apariencia corporal" (Fredrickson y Roberts, 1997, p. 180).

La autocosificación y la autovigilancia corporal, pueden llevar a las mujeres a experimentar emociones negativas, como la vergüenza, ansiedad y reducción de la conciencia de los estados internos corporales (Fredrickson y Roberts, 1997). La TC postula que los episodios de cosificación sexual pueden tener lugar en múltiples contextos y adoptar diferentes formas. Así por ejemplo, pueden darse en los medios de comunicación, en las interacciones interpersonales, en el lugar de trabajo, en ámbitos educativos, etc. En cuanto a las formas, las dos maneras específicas de cosificar a las mujeres son: la evaluación corporal y las aproximaciones explícitas no deseadas (Fredrickson y Roberts, 1997).

La cosificación sexual interpersonal puede ser considerada una forma de discriminación sexista, en la que una mujer es tratada de manera diferente por el hecho de ser mujer (Fredrickson y Roberts, 1997; Landrine, Klonoff, Gibbs, Manning y Lund, 1995), y que tiene como objetivo, minimizar la importancia de los atributos internos de la mujer. La exposición repetida de las mujeres a diferentes niveles de cosificación sexual, tiene una influencia directa en los niveles de autocosificación (Fredrickson, Roberts, Noll, Quinn, y Twenge, 1998; Tiggemann y Slater, 2001) e importante consecuencias para ella. Sin embargo, y pese a su importancia, son escasos los estudios que recogen informes de mujeres sobre su percepción ante los eventos de cosificación a los que la están expuestas. Una posible explicación a esta ausencia de datos, se deba a la escasez de instrumentos de medida. Dado lo reciente del desarrollo de la TC, los instrumentos desarrollados para evaluar la cosificación sexual son aún reducidos, siendo el más adecuado, la Interpersonal Sexual Objectification Scale (Kooze, Tylka, AugustusHorvath y Denchik, 2007) (en proceso de adaptación a la población española) principalmente por dos razones. En primer lugar, porque permite que la mujer se identifique como cosificada sexualmente, sin requerir una autoclasificación previa de dicho evento como sexista (Stangor, Sechrist,y Swim, 1999). Moradi, Dirks y Matteson (2005) insisten en que la cosificación sexual sea integrada dentro del marco de la TC, ya que permite un mejor entendimiento del sufrimiento que podría causar a las mujeres estar expuestas a interacciones que las cosifican sexualmente. $\mathrm{Y}$ en segundo lugar, porque esta medida contiene las dos formas anteriormente mencionadas en que la mujer puede ser cosificada; por un lado la evaluación corporal y por otro, las aproximaciones explícitas no deseadas.

\section{Diferencias de género en eventos interpersonales de cosificación sexual}

En su inicio, la TC se formuló como marco de referencia para entender la socialización en un contexto en el que se valora a la mujer como un instrumento para dar placer. Sin embargo, en nuestros días, estas exigencias respecto al cuerpo, también se han extendido a los hombres (Leit, Pope y Gray, 2001), idealizando sus cuerpos, presentándolos como más musculosos e inalcanzables, lo que conlleva que en la actualidad, los hombres estén menos satisfechos de lo que lo estaban en el pasado (Garner, 1997). Esta nueva realidad nos lleva a plantearnos la posibilidad de que la TC pueda también servir como marco explicativo de la menor satisfacción con el cuerpo que muestran los hombres hoy día y que podamos inferir la posibilidad de que ellos también pueden llegar a autocosificarse (Strelan y Hargreaves, 2005). La investigación empírica ha mostrado que los hombres que tienen mayores puntuaciones en autocosificación, obtienen menores puntuaciones en autoestima (McKinley, 2006).

Sin embargo, no podemos olvidar que la cosifica- 
ción sexual, como una forma más sutil de discriminación sexista (Klonoff y Landrine, 1995), tiene una mayor prevalecía entre mujeres que entre hombres (Swim, Hyers, Cohen y Ferguson, 2001). Desde una perspectiva feminista, esto se explica porque hombres y mujeres construyen socialmente su cuerpo de manera diferente, ya que las mujeres lo construyen para ser mirado (Spitzack, 1990), lo que explica que experimenten menos sentimientos positivos hacia este que los hombres (Cash, Winstead y Janda, 1986).

\section{Sexismo y Cosificación: importancia del Sexismo Benévolo}

Las mujeres están expuestas cotidianamente a eventos sexistas de todo tipo. La literatura muestra que las mujeres están expuestas a más sexismo que los hombres y que experimentan de uno a dos eventos sexistas semanales (Swim et al., 2001).

La Teoría del Sexismo Ambivalente formulada por Glick y Fiske (1996), resalta la idiosincrasia del prejuicio contra las mujeres, debido a los sentimientos positivos que existen hacia ellas y que coexisten con sentimientos negativos, hostiles o de rechazo.

El sexismo benévolo se convierte en una ideología legitimadora del estatus quo incrementando la satisfacción de la mujer con el sistema social (Jost y Kay, 2005), limitando su participación en acciones colectivas en contra de la discriminación sexista (Becker y Wright, 2011), y reduciendo la percepción de determinados comportamientos como sexistas en hombres y mujeres por no tratarse de un comportamiento hostil (Barreto y Ellemers, 2005). Las consecuencias del sexismo benévolo para las mujeres ha sido suficientemente mostrada, tanto en el ámbito de aspiraciones laborales y situaciones de discriminación (Moya, Glick, Expósito, de Lemus y Hart, 2007), como en las relaciones interpersonales, llevándolas a aceptar en mayor medida la discriminación sexista, reforzando las diferencias de poder entre hombres y mujeres (Glick y Fiske, 1996) e incluso aumentando la tolerancia hacia el maltrato (Expósito, Herrera, Moya y Glick, 2010; Lila, Gracia y García, 2010).

El sexismo benévolo aporta a las mujeres determinados beneficios, como son la admiración y la protección por parte de los hombres. Numerosos estudios han mostrado la relación entre recompensas sociales y la belleza física en la mujer (Davis, 1990; Fiske, Bersoff, Borgida, Deaux y Heilman, 1991). El atractivo físico se convierte de este modo en un aspecto que puede facilitar la consecución de ciertos beneficios sociales (Fredrickson y Roberts, 1997), aunque relega el estatus de la mujer a un nivel más bajo respecto a los hombres en la jerarquía social (Jost y Kay, 2005), puesto que la mujer invierte más tiempo y esfuerzo en adecuar su apariencia al ideal cultural (Dion, Dion y Keelan, 1990).

\section{Cosificación, sexismo y disfrute de la sexualización}

Una de las consecuencias que puede tener el sexismo benévolo es la sexualización. La sexualización de la mujer ocurre cuando ésta es tratada como objeto sexual y evaluada en función de sus características físicas (American Psychological Association, 2007). La sexualización tiene cuatro componentes (a) igualar el valor de una persona a su sexualidad (b) la atracción definida como sexualidad (c) la cosificación sexual (d) y la imposición de la sexualidad; siendo necesario uno de estos cuatro aspectos para poder calificar el suceso como sexualizado (American Psychological Association, 2007).

Tradicionalmente se han investigado las consecuencias de la sexualización poniendo el énfasis en lo negativo del proceso (Fredrickson y Roberts, 1997). Sin embargo, recientemente, la investigación ha mostrado que muchas mujeres disfrutan de ser sexualizadas por los hombres, incluso algunas llegan a sentirse empoderadas al ser apreciadas y admiradas sexualmente por ellos (Baumgardner y Richards, 2004; Gleeson y Frith, 2004)

El disfrute de la sexualización ocurre cuando la mujer encuentra la atención sexual basada en su apariencia como positiva y reforzante (Liss, Erchull, Ramsey, 2011). Numerosos estudios apoyan que la sexualización es una forma de controlar a las mujeres (Gill, 2008), mientras que otros defienden que la sexualización es una manera de empoderamiento que hace que las mujeres asuman el control de su sexualidad (Baumgardner y Richards, 2004). Así, las mujeres atractivas sexualmente podrían experimentar consecuencias subjetivamente positivas, debido a la atención sexual que reciben (Snyder, Tanke y Berscheid, 1977). De este modo, la sexualización se convierte en un mecanismo para la atracción, y una manera de obtener las recompensas que se derivan de ajustarse a los estándares de belleza establecidos (Fiske et al., 1991). Muchas mujeres llevan a cabo conductas que pueden denotar que disfrutan resaltando su cuerpo como por ejemplo llevar camisetas ajustadas, mostrar su pecho o tatuarse el logotipo de "play boy", entre otras (Levy, 2005). Aunque no siempre, exhibir este tipo de comportamiento suele tener consecuencias positivas para quien las realiza. Así por ejemplo, Glick, Larsen, Johnson y Branstiter (2005) encontraron que los aspirantes a un puesto de trabajo que llevaban ropa "provocadora" eran valorados como menos competentes que los que llevaban ropa más conservadora. Las mujeres que disfrutan sexualizándose intentan mantener la atención sexual masculina a través de diferentes mecanismos, siendo éste uno de ellos. Los comportamientos de sexualización están muy relacionados con la autocosificación (Nowatzki y Morry, 2009).

Las mujeres que disfrutan sexualizándose informan de mayores experiencias de cosificación, y esto puede deberse a varias razones. Por un lado, es posible que 
puedan participar en comportamientos y situaciones que aumenten la probabilidad de ser cosificadas sexualmente (Liss et al, 2011); y por otro, podrían estar más atentas a dicho proceso porque es reforzante para ellas. Sin embargo, la evidencia empírica que relaciona el sexismo y el disfrute de la sexualización en mujeres arroja datos contradictorios. Si bien algunos resultados han mostrado que las mujeres sexistas benévolas disfrutan más siendo sexualizadas (Liss et al, 2011) y hacen un mayor uso de cosméticos (Franzoi, 2001). Sin embargo, Nowatzki y Morry (2009) encontraron resultados opuestos, señalando que la no relación entre sexismo benévolo y comportamientos de autosexualización, se justifica por la idea ampliamente compartida de que la mujer debe ser pura y virtuosa (idea que contradice la esencia de la sexualización).

\section{Cosificación, poder y autoestima}

El poder ha sido muy estudiado en el campo de la Psicología Social. Se define como la capacidad de influir en los demás (Vescio, Gervais, Snyder y Hoover, 2005), y controlar los resultados de los demás (Fiske, 1993).

En el caso de las mujeres, ¿cómo ellas podrían aumentar el poder que tienen sobre los hombres? La literatura ha mostrado que muchas mujeres jóvenes disfrutan sexualizándose (Lerum y Dworkin, 2009), y esto puede conllevar algún beneficio para ellas. Determinadas perspectivas teóricas señalan el empoderamiento de la mujer a través de la sexualidad denominando a ésta corriente la tercera ola feminista (Baumgardner y Richards, 2004). Si bien algunos autores consideran a este efecto como falso empoderamiento, es necesario diferenciar entre el sentimiento subjetivo de empoderamiento (Lamb y Peterson, en prensa) y el hecho de ser/estar verdaderamente empoderada, puesto que dicho empoderamiento se da en una estructura social en la que la mujer es valorada meramente por su apariencia y únicamente aceptada si ésta se ajusta a los patrones previamente establecidos (American Psychological Association, 2007). Si bien la cosificación puede aumentar el sentimiento de poder en los hombres, otorgándoles mayor oportunidad de elección y mayores exigencias en sus relaciones interpersonales, en las mujeres, asumir la cosificación las puede limitar a un rol de mayor dependencia sexual de los hombres, en definitiva, consolidando aún más su estatus inferior.

Por último, cabría mencionar algunas de las posibles consecuencias que puede tener la cosificación en las personas, concretamente en su autoestima. La relación entre la cosificación sexual y la autoestima ha quedado suficientemente probada. Las personas que experimentan autocosificación, tienen baja autoestima global y baja autoestima corporal (Strelan, Mehaffey y Tiggemann, 2003). Sin embargo, esta relación es diferente en hombres y en mujeres.
En las mujeres, la relación entre autoestima y exposición a cosificación sexual, presenta una doble interpretación. Por un lado, las mujeres poseen una autoestima frágil ya que su valor está directamente vinculado a su apariencia (Crocker, Luhtanen, Cooper y Bouvrette, 2003; Crocker y Wolfe, 2001). Pero por otro, el atractivo sexual puede atenuar e incluso invertir la relación entre autoestima y autocosificación (Sinclair y Myers, 2004). Así, las mujeres que se cosifican y se sienten atractivas, podrían aumentar temporalmente su autoestima aunque a largo plazo, los niveles de autoestima no se mantienen.

Entre los hombres, esta relación dual no se da al no estar expuestos a tantos eventos de cosificación sexual interpersonal (Frith y Gleeson, 2004). A ellos no se les socializa equiparando su valor al de su apariencia física, por lo que prestan menos atención a su físico, vigilando su apariencia en menor medida que las mujeres.

Como ya se ha recogido en párrafos anteriores, la sexualización podría funcionar como un mecanismo de control sobre las mujeres, otorgándoles beneficios por su apariencia a corto plazo, que tiene como consecuencia el que permanezcan en su estatus quo, y colocándolas en situación de mayor vulnerabilidad.

En definitiva, los principales objetivos de este estudio son los siguientes:

1. Analizar el fenómeno de la cosificación sexual interpersonal en una muestra española.

2. Estudiar el efecto del género en la percepción de experiencias de cosificación sexual interpersonal, en sus dos dimensiones: evaluación corporal y avances explícitos no deseadas.

3. Analizar la relación entre la percepción de cosificación sexual interpersonal con variables ideológicas como el sexismo benévolo y la sensación de poder, así como con autoestima y disfrute de la sexualización.

4. Analizar la influencia de la autoestima y el disfrute de la sexualización en la experiencia de cosificación interpersonal en hombres y en mujeres.

\section{Método}

\section{Participantes}

La muestra estuvo compuesta por 251 participantes de población general (153 mujeres y 98 hombres), con edades comprendidas entre 18 y 63 años y una media de $27.59(D T=10.47)$. El mayor porcentaje de participantes habían realizado estudios Universitarios (69.3\%), el 9.6\% habían cursado Bachillerato, un $8.4 \%$ Formación Profesional, el 6.4\% Secundaria, el 5.6\% tenían estudios primarios y tan sólo el $0.4 \%$ no tenían estudios. 


\section{Procedimiento y Diseño}

La muestra fue recogida mediante un muestreo incidental llevado a cabo en las ciudades de Granada y Salamanca. Dos investigadores previamente entrenados solicitaban a los participantes su colaboración informándoles del anonimato de sus respuestas y garantizándoles confidencialidad. Se trata de un estudio de tipo correlacional de acuerdo con la clasificación propuesta por Montero y León (2007).

\section{Instrumentos}

Se construyó un cuestionario que incluía las siguientes medidas:

- Características sociodemográficas. Se recogieron datos relativos al sexo, edad, nivel de estudios, situación laboral, nacionalidad y orientación sexual de los participantes.

- Escala de Cosificación Sexual Interpersonal (In-terpersonal Sexual Objectification Scale, ISOS) (Kooze et al., 2007) (en proceso de adaptación a muestra española por este mismo equipo). Consta de 15 ítems que evalúan la cosificación sexual interpersonal y está compuesta por dos dimensiones: la evaluación del cuerpo (11 ítems) (e.g. “¿Con qué frecuencia has sentido que alguien estaba mirando fijamente tu cuerpo?") y avances sexuales explícitos no deseados (4 ítems) (e.g. "¿Con qué frecuencia te han manoseado contra tu voluntad?"). El formato de respuesta es tipo Likert de 5 alternativas que oscila entre 1 (Nunca) a 5 (Casi siempre). Los primeros datos psicométricos en población española muestran una buena fiabilidad con un coeficiente alfa de Cronbach de la escala total igual a .89 y de .90 y .74 para las subescalas de evaluación del cuerpo y de avances sexuales explícitos no deseados, respectivamente. Estos coeficientes fueron similares a los obtenidos por Kooze et al., (2007) que fue de .92 en la escala total, .91 en la subescala evaluación del cuerpo y de .78 en la subescala avances explícitos no deseados.

- Escala de Autoestima de Rosenberg (EAR) (Rosenberg, 1965). Es un autoinforme que consta de 10 ítems que evalúan el grado de satisfacción que tiene una persona consigo misma. El formato de respuesta es tipo Likert de 1 (Totalmente en desacuerdo) a 4 (Totalmente de acuerdo). La consistencia interna de la versión original oscila entre .76 y .87 , mostrando en el presente estudio un coeficiente alfa de Cronbach igual a .84 .
- Sensación de Poder (Sense of Power Scale) (An-derson y Galinski, 2006). Escala de 8 ítems que miden las creencias generalizadas sobre el poder que las personas tienen con los demás. El formato de respuesta es tipo Likert de 1 (Totalmente en desacuerdo) a 7 (Totalmente de acuerdo). El coeficiente alfa de Cron-bach es igual a .77, ligeramente más bajo al obtenido por los autores de la escala original.

- Escala de Disfrute de la Sexualización (Enjoyment of Sexualization Scale, ESS) (Liss et al., 2011) (en proceso de adaptación a muestra española por este mismo equipo). Es una escala de 8 ítems que evalúa el disfrute de la sexualización en un contexto interpersonal (e.g. "Me gusta sentirme sexy"). El formato de respuesta es tipo Likert de 1 (Totalmente en desacuerdo) a 6 (Totalmente de acuerdo). La consistencia interna en este estudio fue de 0.89 , similar a la obtenida por Liss et al., (2011) que fue igual a .85.

- Inventario sobre Sexismo Ambivalente (Glick y Fiske, 1996; adaptada al castellano por Expósito, Mo-ya y Glick, 1998). Consta de 22 ítems con un formato de respuesta tipo Likert de 0 (Totalmente en desacuerdo) a 5 (Totalmente de acuerdo). El inventario mide dos tipos de sexismo: Sexismo Hostil, compuesto por 11 items (e.g., "las mujeres se ofenden muy fácilmente") y Sexismo Benévolo compuesto por otros 11 ítems (e.g., "las mujeres deben ser queridas y protegidas por los hombres"). Es una medida con una amplia evidencia acerca de la bondad de sus características psicométricas. En el presente trabajo se obtuvo un coeficiente alpha para la escala total igual a .92 , y de .91 y .85 para la subescala de sexismo hostil y de sexismo benévolo respectivamente.

\section{Resultados}

Con el objetivo de analizar si existen diferencias entre hombres y mujeres en la percepción de cosificación sexual interpersonal, se realizó una $t$ de student para muestras independientes (véase Tabla 1).

Los resultados muestran diferencias significativas en todas las medidas analizadas en función del género. Las mujeres obtienen mayores puntuaciones en la percepción de cosificación sexual interpersonal (ISOS) así como mayores puntuaciones en la dimensión de Evaluación del Cuerpo. Sin embargo, en la dimensión Avances Explícitos no Deseados obtienen puntuaciones significativamente inferiores que la muestra de hombres (1.33 vs. 1.48).

Tabla 1. Diferencias de género en la percepción de cosificación sexual interpersonal

\begin{tabular}{lcccc}
\hline & Mujer & Hombre & $t$ & $p$ \\
\hline ISOS & 2.18 & 1.86 & 4.54 & .000 \\
Evaluación Cuerpo & 2.49 & 2.00 & 5.99 & .000 \\
Avances explícitos no Deseados & 1.33 & 1.48 & -2.09 & .037 \\
\hline
\end{tabular}




\section{Relación entre la percepción de cosificación sexual interpersonal y variables ideológicas (poder y sexismo), autoestima y disfrute de la sexualización}

Tal y como se observa en la Tabla 2, las puntuaciones en la cosificación sexual interpersonal (ISOS) correlacionaron positiva y significativamente con el poder, tanto en mujeres $(r=.17, p<.05)$ como en hombres $(r=.35, p<.001)$, de tal forma que los participantes que percibían más situaciones de cosificación sexual interpersonal se sentían con más poder. Las puntuaciones en la ISOS también correlacionaron positiva interpersonal que los hombres. En relación con el poder, una mayor sensación de poder estuvo relacionada con una mayor percepción de cosificación. A su vez, se ha observado una interacción entre el Sexo y el Poder en la percepción de cosificación $t(248)=2,10$, $\beta=.15, p=.037$. En los hombres, cuanto mayor es la percepción de cosificación más altas son las puntuaciones en poder $t(95)=3.66, \beta=.35, p<.001$. En mujeres, si bien el poder también predice la percepción de cosificación interpersonal $t(149)=2.14, \beta=.39, p=$ .037) esta relación no fue tan acusada como en los hombres.

Tabla 2. Correlaciones bivariadas entre las diferentes variables

\begin{tabular}{|c|c|c|c|c|c|c|c|c|c|c|c|c|}
\hline & \multicolumn{2}{|c|}{1} & \multicolumn{2}{|c|}{2} & \multicolumn{2}{|c|}{3} & \multicolumn{2}{|c|}{4} & \multicolumn{2}{|c|}{5} & \multicolumn{2}{|c|}{6} \\
\hline & $M$ & $H$ & $M$ & $H$ & $M$ & $H$ & $M$ & $H$ & $M$ & $H$ & $M$ & $H$ \\
\hline 1. ISOS & - & - & & & & & & & & & & \\
\hline 2. $S H$ & .12 & -.05 & - & - & & & & & & & & \\
\hline 3. $S B$ & $.20 * *$ & .13 & $.56^{* * * *}$ & $.48 * * *$ & - & - & & & & & & \\
\hline 4. Poder & $.17^{*}$ & $.35^{* * *}$ & $-.24 * *$ & .12 & -.13 & -.01 & - & - & & & & \\
\hline 5. EAR & .04 & $.24 *$ & $-.30^{* * * *}$ & .02 & $-.21 * *$ & -.01 & $.61 * * *$ & $.35^{* * * *}$ & - & - & & \\
\hline 6. ESS & $.40 * * *$ & $.35^{* * * *}$ & -.01 & .15 & $.27 * * *$ & .15 & .11 & $.38^{* * * *}$ & .03 & .18 & - & - \\
\hline
\end{tabular}

Nota: ISOS: Cosificación interpersonal; SH: Sexismo Hostil; SB: Sexismo benévolo; Poder: Sensación de poder; EAR: Autoestima; ESS: Disfrute de la sexualización $* p \leq .05 * * p \leq .01 * * * p \leq .001$

y significativamente con el disfrute de la sexualización, de modo que los participantes que se sentían más cosificados sexualmente disfrutaban más sexualizándose (mujeres: $r=.40, p<.001$; hombres: $r=.35, p<.001$ ).

Entre las mujeres, la ISOS obtuvo una correlación positiva y significativa con el sexismo benévolo $(r=$ $.20, p<.01)$, de tal forma que aquellas que mayor cosificación sexual interpersonal percibían obtenían mayores puntuaciones en sexismo benévolo. En los hombres, la ISOS correlacionó positiva y significativamente con la autoestima. Los hombres que se sentían más cosificados, presentaban una mayor autoestima $(r=$ $.24, p<.05)$.

\section{Efecto del Sexo y el Poder en la cosificación sexual interpersonal}

Con el fin de explorar el efecto del sexo del participante y el poder en la cosificación sexual interpersonal, se realizó un análisis de regresión jerárquica. La variable criterio fue la puntuación en la percepción de cosificación sexual interpersonal y como variables predictoras fueron introducidas en el paso 1, el sexo del participante y la puntuación en poder, y en paso 2 la interacción entre el sexo y el poder. Los resultados mostraron efectos principales del Sexo $t(248)=-4.93$, $\beta=-.29, p<.001 \mathrm{y}$ del Poder $t(248)=3.85, \beta=.23$, $p<.001$. Respecto al sexo, las mujeres obtuvieron mayores puntuaciones en percepción de cosificación

\section{El efecto mediador del poder entre la autoestima y la cosificación sexual interpersonal en hombres}

Para analizar el posible papel mediador del poder entre la autoestima y la cosificación sexual interpersonal, se siguieron las recomendaciones de Baron y Kenny (1986). Según los autores, se deben cumplir tres condiciones para mostrar mediación. En primer lugar, que la variable independiente (autoestima) influya en la variable mediadora (poder). Segundo, que la variable mediadora se relacione con la variable dependiente (cosificación sexual interpersonal). Y, en tercer lugar, que exista una relación significativa entre la variable independiente y la dependiente, que una vez introducida la variable mediadora pierda, parcial o totalmente, su efecto. Se comprobaron estos requisitos mediante diversos análisis de regresión. Tal y como se aprecia en la Figura 1, los resultados indicaron que la autoestima influye significativamente en la cosificación sexual interpersonal $(\beta=.24, p=.017)$. Igualmente, mediante un análisis de regresión independiente, se observó una relación significativa entre la autoestima y el poder $(\beta=.35, p<.001)$. Además, en el paso tres de la regresión, la autoestima pierde su efecto sobre la cosificación sexual interpersonal cuando se introduce el poder. El test de Sobel (Sobel, 1982) mostró que la mediación fue significativa $(z=2.28, p<.05)$, por lo que podemos afirmar que el poder media completamente la relación entre la autoestima y la percepción de cosificación sexual interpersonal. 
Figura 1. El papel mediador del poder en la cosificación sexual interpersonal y autoestima en hombres

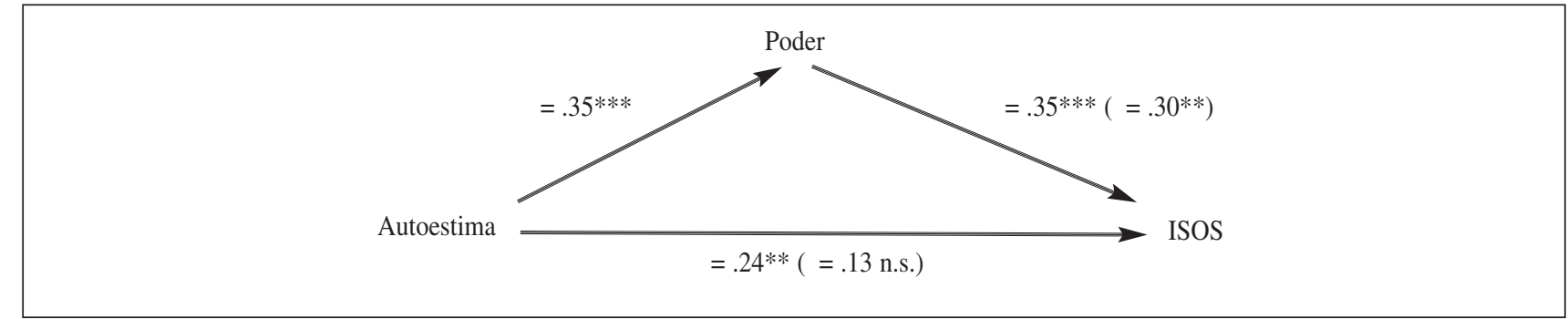

Nota. $* p<.05 * * p<.01 * * * p<.001$

\section{El Disfrute de la Sexualización como mediador entre el poder y la cosificación sexual interpersonal en hombres}

Para analizar el posible papel mediador del disfrute de la sexualización entre el poder percibido y la cosificación sexual interpersonal se siguieron las recomendaciones de Baron y Kenny (1986) anteriormente mencionadas. Tal y como se observa en la Figura 2, los resultados mostraron que la variable poder influye significativamente en la cosificación sexual interpersonal $(\beta=.35, p<.001)$. Igualmente, se observó una relación significativa entre el poder y el disfrute de la sexualización $(\beta=.38, p<.001)$. Además, en el paso tres de la regresión, se observa que el poder pierde parcialmente su efecto sobre la cosificación sexual interpersonal cuando se introduce la variable disfrute de la sexualización. Para examinar si la reducción del efecto del poder sobre la variable dependiente era significativa se realizó el test de Sobel (Sobel, 1982) que indicó que el descenso fue significativo $(z=2.04, p<.05)$. Por lo tanto, de los análisis se desprende que el disfrute de la sexualización media parcialmente la relación entre el poder y la percepción de cosificación sexual interpersonal.

\section{El Disfrute de la Sexualización como mediador entre el sexismo benévolo y la cosificación sexual interpersonal en mujeres}

Otro de los objetivos consistió en analizar el posible efecto mediador del disfrute de la sexualización entre el sexismo benévolo y la cosificación sexual interpersonal. Para ello, se procedió de acuerdo al procedi- miento anteriormente descrito. Como se puede apreciar en la Figura 3, los resultados mostraron que el sexismo benévolo influye significativamente en la cosificación sexual interpersonal $(\beta=.21, p=.01)$. Igualmente, se observó una relación significativa entre el sexismo benévolo y el disfrute de la sexualización $(\beta=.27, p<.001)$, así como que el sexismo benévolo pierde su efecto sobre la cosificación sexual interpersonal cuando se introduce en la regresión la variable disfrute de la sexualización. El test de Sobel (Sobel, 1982) mostró que la mediación fue significativa $(z=2.04, p<.05)$, de lo que se desprende que el disfrute de la sexualización media completamente la relación entre sexismo benévolo y la percepción de cosificación sexual interpersonal.

\section{Discusión}

El mundo en el que vivimos es un mundo de hombres, mayoritariamente heterosexual, que potencialmente cosifica el cuerpo de la mujer (Vaes, Paladino y Puvia, 2011), por ello este estudio intenta indagar el fenómeno de la cosificación sexual interpersonal y las variables que operan en el mismo.

Uno de los objetivos principales consistió en analizar el fenómeno de la cosificación sexual interpersonal en hombres y en mujeres. Los resultados han mostrado que las mujeres perciben una mayor cosificación sexual en sus relaciones interpersonales. Este dato queda avalado por estudios previos que demuestran que las mujeres experimentan más cosificación sexual que los hombres (Plous y Neptune, 1997). En concreto, se ha demostrado que las mujeres experimentan el

Figura 2. El papel mediador del disfrute de la sexualización en el poder y en la cosificación sexual interpersonal en hombres

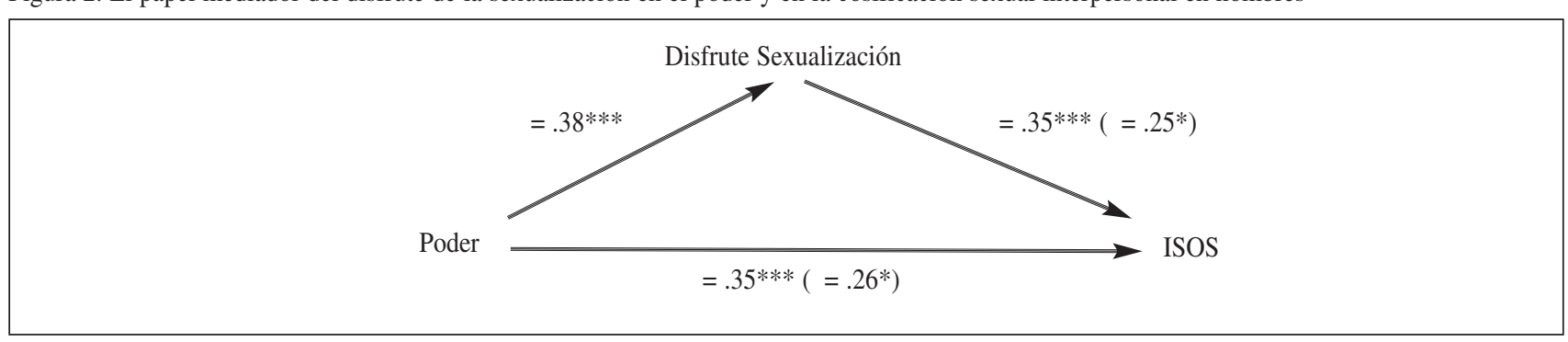

Nota. $* p<.05 * * p<.01 * * * p<.001$ 
Figura 3. El papel mediador del disfrute de la sexualización en el sexismo benévolo y en la cosificación sexual interpersonal en mujeres

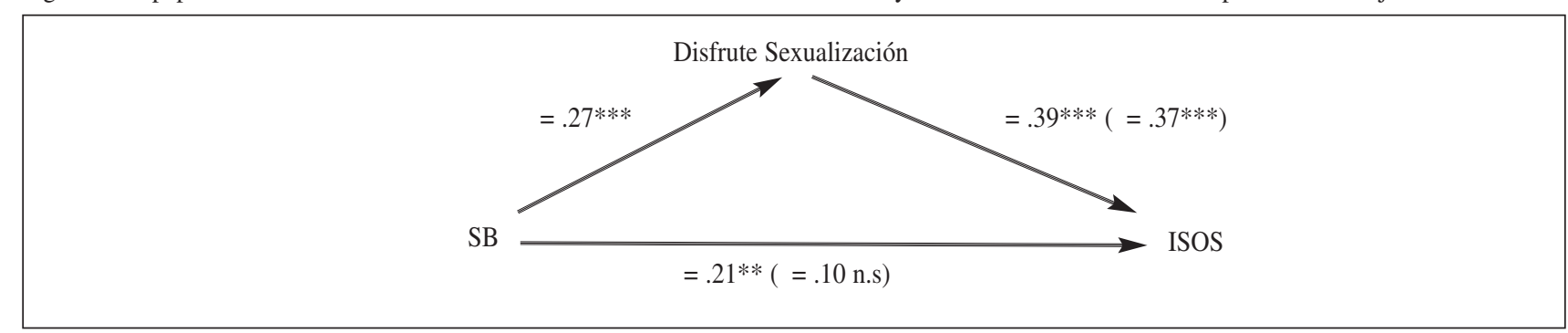

Nota. $* p<.05 * * p<.01 * * * p<.001$

doble de eventos sexistas semanales que los hombres (Swim et al., 2001).

Si se analizan las dos dimensiones de la escala de experiencias de cosificación sexual interpersonal (ISOS), las mujeres obtienen mayores puntuaciones en la dimensión de evaluación corporal pero obtienen una puntuación menor en la evaluación de avances explícitos no deseados. Este resultado, sorprendente, puede ser debido a varias razones, una posible, puede ser que las mujeres no son conscientes de muchos actos sexistas a los que están expuestas, otra, el que hayan llegado incluso a normalizar muchas de esas situaciones y una tercera, puede estar relacionada con el desconocimiento de la intención del perpetrador (Swim, Scott, Sechrist, Campbell y Stangor, 2003). Según Becker y Swim (2011) aumentar la conciencia de los eventos sexistas más sutiles en las mujeres, puede resultar en una manera de intervención eficaz para reducir el sexismo del que son objeto.

El segundo de los objetivos perseguidos consistió en explorar la relación entre variables ideológicas, autoestima y disfrute de la sexualización. Los resultados mostraron relaciones significativas entre determinadas variables ideológicas y la percepción de cosificación sexual, y además se observaron diferencias de género en la relación entre las variables. Concretamente, una de las variables ideológicas analizadas, el poder, se relacionó con una mayor percepción de cosificación, pero ésta relación fue más acusada en hombres que en mujeres. Este dato es consistente con investigaciones previas, que afirman que el poder mantiene una relación directa con la cosificación (Bargh, Raymond, Pryor y Strack, 1995) y de un modo más específico, que las diferencias de poder intervienen en la cosificación de las personas, lo que podría explicar un uso diferencial de la cosificación por ambos sexos (Brewer, 1982).

Pese a la intrumentalización que sufren las mujeres cuando se sienten cosificadas sexualmente, muchas de ellas se sienten empoderadas, aunque se trate de un falso empoderamiento (Lerum y Dworkin, 2009), en la medida en les otorga un sentimiento ilusorio de autonomía, mientras que a su vez siguen inmersas en una estructura social patriarcal en la que las mujeres siguen siendo valoradas por su apariencia (American Psychological Association, 2007).
Otra de las variables de interés fue la autoestima y su relación con la cosificación. Los resultados han mostrado la relación entre la autoestima y las experiencias de cosificación sexual en hombres, pero no en mujeres. Como era de esperar, las mujeres no ven reforzada su autoestima al ser cosificadas sexualmente. Este resultado es congruente con la noción de "descontento normativo" (Rodin, Silberstein y StriegelMoore, 1985). Puesto que las mujeres sienten una insatisfacción corporal generalizada debido al hecho de haber interiorizado el ideal corporal femenino como parte esencial de su autoconcepto (Crocker y Wolfe, 2001), cuando éste ideal corporal se percibe inalcanzable, la discrepancia entre el ideal y la realidad, provoca malestar y repercute en su autoconcepto y autoestima (Rodríguez, Goñi y Ruiz de Azúa, 2006).

Sin embargo en hombres, resultó interesante el efecto mediador del poder entre la autoestima y la percepción de cosificación sexual interpersonal. Como se menciona en el párrafo anterior, la autoestima de las mujeres no aumenta al ser percibidas como objetos sexuales, ya que se trata de un falso empoderamiento (Lamb y Peterson, en prensa) y la sexualización femenina, una forma de control. Sin embargo, la sexualización masculina no tiene el mismo fin, no se aumenta el valor de la apariencia física masculina para mantenerlos sometidos a una posición subordinada. En el caso de los hombres, la sensación de poder que sienten al ser sexualizados y cosificados, es contingente con su poder real y por lo tanto, tiene efectos positivos en su autoestima.

Respecto al sexismo benévolo, los resultados corroboran investigaciones previas en el sentido de que, las mujeres que tienen mayores puntuaciones en sexismo benévolo experimentan mayores experiencias de cosificación. El sexismo benévolo se erige de nuevo como un arma de subordinación eficaz porque las mujeres están expuestas en mayor medida y son menos capaces de reconocerlo, y por tanto, de luchar contra él (Jackman, 1994). Los resultados encontrados en este estudio son coherentes con los hallados por otros autores en cuanto a que de alguna manera, mayores puntuaciones en sexismo benévolo se relaciona con un mayor uso de cosméticos (Franzoi, 2001) y una mayor interiorización del ideal de delgadez (Forbes, Collinsworth, Jobe, Braun y Wise, 2007), aspectos ambos esenciales que podrían favorecer experiencias de cosificación, y que contribu- 
yen a que las mujeres que asumen esta ideología sexista la perpetúe intentando asumir cualidades típicamente femeninas (Glick y Fiske, 1996)

Por último, uno de los objetivos más relevantes para la investigación consistió en analizar el efecto del disfrute de la sexualización en la percepción de cosificación sexual. Los resultados mostraron que tanto en hombres como en mujeres, un mayor disfrute de la sexualización estaba relacionado con mayores experiencias de cosificación sexual (Liss y Erchull, 2010). Sin embargo, el disfrute de la sexualización tiene un efecto mediador diferencial en función del género. En hombres, el disfrute de la sexualización media la relación entre el poder y la cosificación sexual. De modo que, sentirse poderoso se relaciona con un mayor disfrute de la sexualización, lo que se traduce en una mayor percepción de cosificación sexual. En mujeres, el sexismo benévolo de las participantes activa el disfrute de la sexualización, lo que se traduce en una mayor percepción de experiencias de cosificación (Liss et al., 2011). Disfrutar sexualizándose es compatible con mantener actitudes sexistas que incluyan valorar a la mujer por su apariencia física y por su sexualidad. Las mujeres que disfrutan sexualizándose son aquellas que tienen puntuaciones más altas en sexismo benévolo, y que por lo tanto considera que las mujeres han de ser veneradas y protegidas por los hombres, por lo que cuando un hombre las admira, se sienten alagadas y disfrutan con ello. Estos resultados tienen gran importancia, puesto que conocer que el disfrute de la sexualización es activado por una ideología sexista benévola en las mujeres, conlleva una falsa sensación de poder que camufla la subordinación de la mujer a una posición de inferioridad respecto al hombre y que le permite obtener beneficios efímeros.

A modo de conclusión, los resultados nos hacen reflexionar sobre el fin diferencial de la cosificación sexual femenina y masculina. Todo parece señalar que la cosificación masculina podría producir las mismas consecuencias que la femenina a largo plazo (McKinley, 2006) debido a imposición de los estándares de belleza, pero esconden realidades distintas. En el caso de los hombres, ajustarse a dichos estándares conlleva recompensas sociales inmediatas y reales (aumenta su autoestima y su poder), mientras que las recompensas sociales que otorga la sociedad a las mujeres por cosificarse y ajustarse a los patrones establecidos socialmente, hace que mantengan ideas de subordinación femenina, aumentando el sexismo benévolo y debilitando la acción colectiva de las mujeres contra el patriarcado.

\section{Nota del autor}

Este trabajo ha sido posible gracias a la financiación concedida por el Ministerio de Educación y Ciencia al proyecto de I+D "Ideología sexista y diferencias de poder en el origen y mantenimiento del acoso sexual" (Ref. PSI2011-29720).

\section{Referencias}

Anderson, C. y Galinsky, A. D. (2006). Power, optimism, and risk-taking. European Journal of Social Psychology, 36, 511-536.

American Psychological Association, Task Force on the Sexualization of Girls. (2007). Report of the APA Task Force on the Sexualization of Girls. Washington, DC: Author.

Bargh, J. A., Raymond, P., Pryor, J. B. y Strack, F. (1995). Attractiveness of the underling: An automatic power-sex association and its consequences for sexual harassment and aggression. Journal of Personality and Social Psychology, 68, 768-781.

Baron, R. M. y Kenny, D. A. (1986). The moderatormediator variable distinction in social psychological research: Conceptual, strategic and statistical considerations. Journal of Personality and Social Psychology, 51, 1173-1182.

Barreto, M. y Ellemers, N. (2005). The burden of benevolent sexism: How it contributes to the maintenance of gender inequalities. European Journal of Social Psychology, 35, 633-642.

Bartky, S. L. (1990). Femininity and domination: Studies in the phenomenology of oppression. New York: Routledge.

Baumgardner, J. y Richards, A. (2004). Feminism and femininity: Or how we learned to stop worrying and love the thong. In A. Harris (Ed.), All about the girl: Culture, power, and identity (pp. 59-67). New York, NY: Routledge.

Becker, J. C. y Swim, J. K. (2011). Seeing the unseen: Attention to daily encounters with sexism as way to reduce sexist beliefs. Psychology of Women Quarterly, 35, 227-242.

Becker, J. C. y Wright, S. C. (2011). Yet another dark side of chivalry: Benevolent sexism undermines and hostile sexism motivates collective action for social change. Journal of Personality and Social Psychology, 101, 62-77.

Brewer, M. B. (1982). Further beyond nine to five: An integration and futures directions. Journal of Social Issues, 38, 149-158.

Cash, T. J., Winstead, B. A. y Janda, L. H. (1986). The great American shape-up. Psychology Today, 20, 30-37.

Crocker, J., Luhtanen, R. K., Cooper, M. L. y Bouvrette, A. (2003). Contingencies of self-worth in college students: Theory and measurement. Journal of Personality and Social Psychology, 85, 94-908.

Crocker, J. y Wolfe, C. T. (2001). Contingencies of self-worth. Psychological Review, 108, 593-623.

Davis, S. (1990). Men as success objects and women as sex objects: A study of personal advertisements. Sex Roles, 23, 43-50.

Dion, K. L., Dion, K. K. y Keelan, P. (1990). Appearance anxiety as a dimension of social-evalu- 
ative anxiety: Exploring the ugly duckling syndrome. Contemporary Social Psychology, 14, 220224.

Expósito, F., Herrera, M. C., Moya, M. y Glick, P. (2010). Don't rock the boat: Women's benevolent sexism predicts fears of marital violence. Psychology of Women Quarterly, 34, 20-26.

Expósito, F., Moya, M. y Glick, P. (1998) Sexismo ambivalente: medición y correlatos. Revista de Psicología Social, 13, 159-170.

Fiske, S. T. (1993). Controlling other people - the impact of power on stereotyping. American Psychologist, 48, 621-628.

Fiske, S. T., Bersoff, D. N., Borgida, E., Deaux, K. y Heilman, M. E. (1991). Social science research on trial: Use of sex stereotyping research in Price Waterhouse v. Hopkins. American Psychologist, 46, 1049-1060.

Forbes, G. B., Collinsworth, L. L., Jobe, R. L., Braun, K. D. y Wise, L. M. (2007). Sexism, hostility toward women, and endorsement of beauty ideals and practices: Are beauty ideals associated with oppressive beliefs? Sex Roles, 56, 265-273.

Franzoi, S. L. (2001). Is female body esteem shaped by benevolent sexism? Sex Roles, 44, 177-188.

Fredrickson, B. L. y Roberts, T. A. (1997). Objectification theory: Toward understanding women's lived experiences and mental health risks. Psychology of Women Quarterly, 21, 173-206.

Fredrickson, B. L., Roberts, T., Noll, S. M., Quinn, D. M. y Twenge, J. M. (1998). That swimsuit becomes you: Sex differences in self-objectification, restrained eating, and math performance. Journal of Personality and Social Psychology, 75, 269-284.

Frith, H. y Gleeson, K. (2004). Clothing and embodiment: Men managing body image and appearance. Psychology of Men and Masculinity, 5, 40-48.

Garner, D.M. (1997). The 1997 body image survey results. Psychology Today, 30, 30- 41.

Gleeson, K. y Frith, H. (2004). Pretty in pink: Young women presenting mature sexual identities. In A. Harris (Ed.), All about the girl: Culture, power, and identity (pp. 103-113). New York, NY: Routledge.

Gill, R. (2008). Empowerment/sexism: Figuring female sexual agency in contemporary advertising. Feminism and Psychology, 18, 35-60.

Glick, P. y Fiske, S. T. (1996). The Ambivalent Sexism Inventory: Differentiating hostile and benevolent sexism. Journal of Personality and Social Psychology, 70, 491-512.

Glick, P., Larsen, S., Johnson, C. y Branstiter, H. (2005). Evaluations of sexy women in low- and high-status jobs. Psychology of Women Quarterly, 29, 389-395.

Jackman, M. R. (1994). The velvet glove: Paternalism and conflict in gender, class, and race relations. Berkeley: University of California Press.

Jost, J. T. y Kay, A. (2005). Exposure to benevolent sexism and complementary gender stereotypes: Consequences for specific and diffuse forms of system justification. Journal of Personality and Social Psychology, 88, 498-509.

Kaschak, E. (1992). Engendered lives: A new psychology of women's experience. New York: Basic Books.

Klonoff, E.A. y Landrine, H. (1995). The schedule of sexist events: A measure of lifetime and recent sexist discrimination in women's lives. Psychology of Women Quarterly, 19, 439-472.

Kozee, H. B., Tylka, T. L., Augustus-Horvath, C. L. y Denchik, A. (2007). Development and psychometric evaluation of the Interpersonal Sexual Objectification Scale. Psychology of Women Quarterly, 31, 176-189.

Landrine, H., Klonoff, E.A., Gibbs, J., Manning, V. y Lund, M. (1995). Physical and psychiatric correlates of gender discrimination: An application of the Schedule of Sexist Events. Psychology of Women Quarterly, 19, 473-492.

Lamb, S. y Peterson, Z. (en prensa). Adolescent girls' sexual empowerment: Two feminists explore the concept. Sex Roles.

Leit, R. A., Pope, H. G. y Gray, J. J. (2001). Cultural expectations of muscularity in men: The evolution of playgirl centerfolds. International Journal of Eating Disorders, 29, 90-93.

Lerum, K. y Dworkin, S. L. (2009). "Bad girls rule": An interdisciplinary feminist commentary on the report of the APA Task Force on the Sexualization of Girls. Journal of Sex Research, 46, 250-263.

Levy, A. (2005). Female chauvinist pigs: Women and the rise of raunch culture. New York, NY: Free Press.

Lila, M., Gracia, E. y García, F. (2010) Actitudes de la policía ante la intervención en casos de violencia contra la mujer en las relaciones de pareja: influencia del sexismo y la empatía. Revista de Psicología Social, 25, 313-323.

Liss, M. y Erchull, M. J. (2010). Everyone feels empowered: Understanding feminist self-labeling. Psychology of Women Quarterly, 34, 85-96.

Liss, M., Erchull, M. J. y Ramsey, L. R. (2011). "Empowering or Oppressing? Development and exploration of the Enjoyment of Sexualization Scale." Personality and Social Psychology Bulletin, 37, 55-68.

McKinley, N. M. (2006). Longitudinal gender differences in objectified body consciousness and weightrelated attitudes and behaviors: Cultural and developmental contexts in the transition from college. Sex Roles, 54, 159-173.

Montero, I. y León, O. G. (2007). A guide for naming research studies in Psychology. International Journal of Clinical and Health Psychology, 7, 847862.

Moradi, B., Dirks, D. y Matteson, A. V. (2005). Roles 
of sexual objectification experiences and internalization of standards of beauty in eating disorder symptomatology: A test and extension of objectification theory. Journal of Counseling Psychology, $52,420-428$.

Moya, M., Glick, P., Expósito, F., De Lemus, S. y Hart, J. (2007). It's for your own good: Benevolent sexism and women's reactions to protectively justified restrictions. Personality and Social Psychology Bulletin, 33, 1421-1434.

Nowatzki, J. y Morry, M. (2009). Women's intentions regarding, and acceptance of, self-sexualizing behavior. Psychology of Women Quarterly, 33, 95107.

Plous, S. y Neptune, D. (1997). Racial and gender biases in magazine advertising: A content-analytic study. Psychology of Women Quarterly, 21, 627644.

Rodin, J., Silberstein, L. y Striegel-Moore, R. (1985). Women and weight: A normative discontent. En T. Sonderegger (Ed.), Nebraska Symposium on Motivation: Vol. 32. Psychology and gender (pp. 267-307). Lincoln: University of Nebraska Press.

Rodríguez, A., Goñi, A. y Ruiz de Azúa, S. (2006). Autoconcepto físico y estilos de vida en la adolescencia. Intervención Psicosocial, 15, 81-94.

Rosenberg, M. (1965). Society and the Adolescent Self Image. Princeton: Princeton University Press.

Sevillano, S., Rodríguez, J., Olarte, M. y Lahoz, L. (2005). El conocimiento del pasado. Una herramienta para la igualdad. Salamanca: Plaza Universitaria Ediciones.

Sinclair, S. L. y Myers, J. E. (2004). The relationship between objectified body consciousness and wellness in a group of college women. Journal of College Counseling, 7, 159-161.

Snyder, M., Tanke, E. D. y Berscheid, E. (1977). Social perception and interpersonal behavior: On the selffulfilling nature of social stereotypes. Journal of Personality and Social Psychology, 35, 656-666.

Sobel, M. E. (1982). Asymptotic confidence intervals for indirect effects in structural equations models. En S. Leinhart (Ed.), Sociological Methodology (pp. 290-312). San Francisco: Jossey-Bass.
Sociedad Española de Cirugía Plástica, Reparadora y Estética (2006). España, primer país europeo en operaciones de estética y cuarto del mundo. Recuperado de http://www.consumer.es/-web/es/salud/2006/05/09/151808.php

Spitzack, C. (1990). Confessing excess: Women and the politics of body reduction. Albany: State University of New York Press.

Strelan, P., Mehaffey, S. J. y Tiggemann, M. (2003). Self-objectification and esteem in young women: The mediating role of reasons for exercise. Sex Roles, 48, 89-95.

Strelan, P. y Hargreaves, D. (2005). Reasons for exercise and body esteem: Men's responses to selfobjectification. Sex Roles, 53, 495-503.

Stangor, C., Sechrist, G. B. y Swim, J. K. (1999). Sensitivity to sexism and perceptions of reports about sexist events. Swiss Journal of Psychology, $58,251-256$.

Swim, J. K., Hyers, L. L., Cohen, L. L. y Ferguson, M. J. (2001). Everyday sexism: Evidence for its incidence, nature, and psychological impact from three daily diary studies. Journal of Social Issues, 57, 31-53.

Swim, J., Scott, E., Sechrist, G., Campbell, B. y Stangor, C. (2003). The role of intent and harm in judgments of prejudice and discrimination. Journal of Personality and Social Psychology, 84, 944-959.

Tiggemann, M. y Slater, A. (2001). A test of objectification theory in former dancers and non-dancers. Psychology of Women Quarterly, 25, 57-64.

Vaes, J., Paladino, M. P. y Puvia, E. (2011). Are sexualized females complete human beings? Why males and females dehumanize sexually objectified women. European Journal of Social Psychology, 41, 774-785.

Vescio, T. K., Gervais, S. J., Snyder, M. y Hoover, A. (2005). Power and the creation of patronizing environments: The stereotype-based behaviors of the powerful and their effects on female performance in masculine domains. Journal of Personality and Social Psychology, 88, 658-672.

Wolf, N. (1991). The beauty myth: How images of beauty are used against women. New York: Harper Collins.
Manuscrito recibido: 21/06/2011

Revisión recibida: 28/11/2011

Manuscrito aceptado: 01/12/2011 